\title{
Abhängigkeitserkrankungen und Elternschaft - Herausforderungen und Möglichkeiten der Hilfe
}

\section{Substance Use Disorders and Parenthood - Challenges and Interventions}

\author{
Autoren \\ Diana Moesgen, Michael Klein, Janina Dyba
}

Institut

Deutsches Institut für Sucht- und Präventionsforschung, Katholische Hochschule NRW, Abt. Köln

Schlüsselwörter

Abhängigkeit, Elternschaft, Erziehung, Familie, Kinder

Key words

substance use Disorders, parenthood, parenting, family, children

Bibliografie

DOI http://dx.doi.org/10.1055/s-0043-103060

Suchttherapie 2017; 18: 65-72

(c) Georg Thieme Verlag KG Stuttgart · New York ISSN 1439-9903

Korrespondenzadresse

Dr. Diana Moesgen

Deutsches Institut für Sucht- und Präventionsforschung

Katholische Hochschule NRW

Wörthstraße 10

50668 Köln

d.moesgen@katho-nrw.de

\section{ZUSAMMENFASSUNG}

Kinder aus suchtbelasteten Familien sind einer Vielzahl an belastenden Lebensbedingungen ausgesetzt. Einen besonderen Risikofaktor stellt das problematische Erziehungsverhalten dar, welche suchtkranke Eltern infolge ihrer Abhängigkeitserkrankung oftmals aufzeigen wie z. B. Vernachlässigung oder andere dysfunktionale Erziehungspraktiken. In der Konsequenz können sich bei den Kindern internalisierende oder externalisierende Verhaltensauffälligkeiten oder eigene substanzbezogene Probleme entwickeln. Trotz der dringenden Relevanz gibt es im deutschsprachigen Raum kaum entsprechende Konzepte zur Förderung der Erziehungskompetenz, die sich direkt an suchtkranke Eltern richten. Im US-amerikanischen Raum hingegen existieren verschiedene verhaltens- und bindungsorientierte Elterntrainings für suchtkranke Eltern, die sich als wirksam erwiesen haben.

\section{ABSTRACT}

Children from substance-involved families are exposed to various stressors in their family life. Especially problematic parenting behavior as a result of the parental substance use, like e. $\mathrm{g}$. neglect and other dysfunctional parenting practices, pose a great developmental risk factor for these children. As a consequence, children frequently develop externalizing and internalizing symptoms or own substance-related problems. Despite their relevance, parenting trainings for substance-abusing parents in particular are scarce in Germany. However, there are several US-American behavioral and attachment based programs for substance-abusing parents that have been proven effective.

\section{Epidemiologie}

In Deutschland sind älteren Zahlen zufolge etwa 5-6 Millionen Kinder und Jugendliche unter 20 Jahren von einer elterlichen Alkoholabhängigkeit betroffen [1]. Dies kann auf einer Exposition im Elternhaus gegenüber einem oder zwei suchtkranken Elternteilen beruhen, wobei die Dauer dieser Exposition durchaus variiert. Neuere Hochrechnungen zeigen, dass $22 \%$ der Elternteile, die mit min- destens einem eigenen minderjährigen Kind im Haushalt leben, einen riskanten Alkoholkonsum aufweisen [2]. Dies bedeutet, dass in Deutschland bis zu 6,6 Millionen Kinder mit einem Elternteil mit riskantem Alkoholkonsum zusammenleben. In Bezug auf eine elterliche Drogenabhängigkeit besagen Schätzungen, dass etwa 60000 Kinder von einem opiatabhängigen Elternteil abstammen und mit diesem zusammenleben [3]. 


\section{Risikofaktoren für Kinder aus suchtbelasteten Familien}

\section{Pränatale Exposition}

Bereits während der Schwangerschaft können Kinder im Mutterleib den toxischen Folgen von Alkohol- und Drogenkonsum ausgesetzt sein. Generell birgt eine pränatale Exposition von derartigen Substanzen die Gefahr eines geringen Geburtsgewichts, früher Fütterungsstörungen, einer erhöhten Irritabilität der Neugeborenen sowie eine verzögerte kognitive oder körperliche Entwicklung [4]. Speziell in Bezug auf eine pränatale Alkoholexposition besteht für das ungeborene Kind außerdem die Gefahr der Entwicklung eines Fetalen Alkoholsyndroms (FAS), welches sich durch ein geringes Geburtsgewicht, eine geringe Körpergröße bei Geburt, facialen Auffälligkeiten und Schädigungen des Zentralen Nervensystems auszeichnet [5]. Methamphetaminmissbrauch in der Schwangerschaft ist im Allgemeinen assoziiert mit Aborten und Frühgeburten sowie mit einer zu geringen Körpergröße gemäß Gestationsalter, niedrigem Erregungsniveau, Bewegungseinschränkungen, erhöhtem physiologischen Stress sowie späteren Verhaltens- und Entwicklungsdefiziten [6]. Darüber hinaus kann ein pränataler Methamphetaminmissbrauch, sowie noch stärker ein pränataler Opioidmissbrauch, infolge der beendeten Zufuhr der Substanz im Mutterleib nach der Geburt zu einem Neonatalen Abstinenzsyndrom (NAS) führen [4]. Probleme durch eine pränatale Exposition an Suchtmittel können sich jedoch auch noch später manifestieren: Im Fall von Kokain bspw. in Form von Sprachentwicklungsstörungen, emotionalen Problemen und Verhaltensauffälligkeiten im späteren Kindesalter [7]. Diese pränatal erworbenen Schädigungen interagieren oft in komplexer Weise mit weiteren ungünstigen, postnatalen Entwicklungsbedingungen (s.u.).

\section{Psychosoziale Lebensbedingungen suchtbelasteter Familien}

Sozioökonomische Benachteiligungen Sozioökonomische Benachteiligungen gehören zu den typischen Stressoren für Kinder aus suchtbelasteten Familien, da die materiellen und finanziellen Bedingungen in suchtbelasteten Familien oftmals schlechter sind als in unbelasteten Familien, z. B. durch Arbeitslosigkeit und Verschuldung als Konsequenz des Suchtproblems [8]. Der soziale Abstieg der Erwachsenen im Zuge der Suchterkrankung betrifft insofern auch die Kinder, da diese früh von gesellschaftlicher Ausgrenzung bedroht werden. Auch soziale Marginalisierung, Exklusion und Stigmatisierung können auftreten.

Diskontinuität der Eltern-Kind-Beziehung Einen weiteren Risikofaktor stellt die Diskontinuität und Instabilität der Beziehung zwischen Eltern und Kindern in suchtbelasteten Familiensystemen dar. Durch z. B. Trennungen und Scheidungen der Eltern oder sogar den Tod eines Elternteils sowie durch Fremdunterbringungen der Kinder, stationäre Aufenthalte oder Inhaftierungen der Eltern entstehen wiederholt Beziehungsabbrüche. Eine Studie zu elterlicher Methamphetaminabhängigkeit zeigte, dass über ein Drittel der Kinder von Klienten der ambulanten Suchtberatung in Fremdunterbringung lebte [9].

\section{Elterliches Verhalten in Kontext von Substanzkon- sum und Abhängigkeitserkrankungen}

Suchtkranke Eltern können genauso wie andere Eltern funktionales wie auch dysfunktionales Verhalten zeigen. In Bezug auf ihre Elternrolle und die resultierenden Erziehungsaufgaben ist ein erhöhtes Risiko für dysfunktionale Verhaltensweisen festzustellen $[9,10]$. Dieses kann vor allem aus weniger Zuwendung und Wärme, mehr Aggressivität und Gewalt sowie weniger kontinuierlichen und sicheren Bindungsverhaltensweisen bestehen. Elternkompetenz stellt die Gesamtheit aller Fähigkeiten dar, einem schutzbefohlenen Kind entwicklungsförderliche, sichere Bedingungen und Interaktionen bereitzustellen, sodass dieses sich vor dem Hintergrund seiner Anlagen und Fähigkeiten optimal entwickeln kann. Sie ist nicht identisch mit dem Vermeiden von Stress und Krisen im Leben des Kindes. Nur dann kann ein Kind auch angemessene Resilienzen und Stressbewältigungskompetenzen entwickeln. Erziehungskompetenz basiert auf einer sicheren Rollenkompetenz der Eltern, ihrer psychischen Stabilität und einer liebevollen, altersangemessenen Zugewandtheit zum Kind. Dabei ist es wichtig, dass sich Freiheit und Grenzen in der Erziehung des Kindes in einer entwicklungsförderlichen, aber auch schützenden Balance befinden. Als zentrale Bestandteile der Elternkompetenz sind (1) Beziehungsfähigkeit, (2) Interaktions- und Kommunikationsfähigkeit, (3) Grenzsetzungsfähigkeit, (4) Förder- und Verstärkungsfähigkeit, (5) Vorbildfähigkeit und (6) Alltagsmanagementfähigkeit anzusehen [11].

Vernachlässigung Kinder aus suchtbelasteten Familien sind neben den schwierigen sozioökonomischen Bedingungen auch verschiedenen psychosozialen Belastungen ausgesetzt, die zunächst unabhängig von der Art bzw. des Wirkspektrums der konsumierten Substanz sind. Hierzu gehören z. B. die typischen, substanzübergreifenden Charakteristika einer Abhängigkeitserkrankung wie z. B. Entzugssymptome oder eine Einengung des Elternteils auf den Substanzgebrauch und eine daraus resultierende Vernachlässigung anderer Aktivitäten. Dies führt häufig zu einer Vernachlässigung der Versorgung des Kindes, sowohl auf materieller als auch emotionaler Ebene [9].

Zusätzlich bestehen differenzielle Risiken abhängig vom Wirkspektrums einer Substanz, wie z. B. Aggressivität, Affektlabilität nach Alkoholkonsum; Apathie, Sedierung bei Opioidintoxikation; oder Agitiertheit und „Punding“ (repetitive/stereotype Handlungen, z. B. zwanghaft anmutendes Sortieren von Gegenständen, Mitesser ausdrücken oder Putzen) bei Methamphetaminkonsum. Diese Intoxikationserscheinungen bestimmen das elterliche Verhalten maßgeblich mit und erzeugen einen ungünstigen Fokus der elterlichen Aufmerksamkeit. Eine regelmäßige Exposition an elterlichen Konsum und Intoxikation sind außerdem insofern kritisch, als dass sie die (positiven) Wirkungserwartungen des Kindes bezüglich der Substanz determinieren und so als ein Faktor den Weg einer familiären Transmission der Suchterkrankung ebnen können.

\section{Anderes problematisches Eltern- und Erziehungsverhalten}

Verschiedene Studien rücken das häufig problematische Erziehungsverhalten (wie z. B. ein unangemessen scharfer Tonfall) der Eltern in den Vordergrund $[4,10]$. Weitere Befunde zeigen, dass innerhalb suchtbelasteter Familien Konflikte oftmals nicht nur verbal ausgetragen werden, sondern auch körperliche Gewalt be- 
- Tab. 1 Auswirkungen des elterlichen Substanzkonsums auf die Erziehungskompetenz.

\begin{tabular}{|l|l|l|l|}
\hline Kontextbezogene Risken & $\begin{array}{l}\text { Selbstbezogene Kompetenzen der } \\
\text { Eltern }\end{array}$ & $\begin{array}{l}\text { Kindbezogene Kompetenzen } \\
\text { der Eltern }\end{array}$ & $\begin{array}{l}\text { Handlungsbezogene } \\
\text { Kompetenzen der Eltern }\end{array}$ \\
\hline $\begin{array}{l}\text { Prekäre Soziodemografische } \\
\text { Bedingungen }\end{array}$ & $\begin{array}{l}\text { Sprunghafte Veränderung von } \\
\text { Stimmung, Verhalten und Vereinba- } \\
\text { rungen }\end{array}$ & $\begin{array}{l}\text { Geringe Sensitivität für kindliche } \\
\text { Bedürfnisse } \\
\text { Geringe emotionale Responsivität }\end{array}$ & $\begin{array}{l}\text { Physische/emotionale } \\
\text { Vernachlässigung }\end{array}$ \\
\hline $\begin{array}{l}\text { Instabilität und Diskontinuität in } \\
\text { der Eltern Kind-Beziehungäquate Verteilung } \\
\text { familiärer Aufgaben }\end{array}$ \\
\hline $\begin{array}{l}\text { Trennung von der Familie } \\
\text { Exposition an ungünstige } \\
\text { Lebensbedingungen der } \\
\text { Drogensubkultur }\end{array}$ & $\begin{array}{l}\text { Git Tendenzen zu emotionaler Kälte, } \\
\text { Reizbarkeit, Gewalt }\end{array}$ & $\begin{array}{l}\text { Kinder } \\
\text { Geringe Förderung von } \\
\text { Handlungskompetenzen der } \\
\text { Kinder }\end{array}$ & „Parentifizierung“ \\
\hline
\end{tabular}

inhalten [12]. Dabei sind die Kinder entweder selbst als Opfer oder als Zeuge involviert [9].

Ein sprunghaft wechselndes Erziehungsverhalten durch die Eltern sowie eine ausgeprägte Stimmungslabilität durch den Substanzkonsum konnte in suchtbelasteten Familien beobachtet werden und gehört zu einer der wichtigsten Konsequenzen der Suchtstörungen auf das familiäre Umfeld [13]. Dies kann sich z. B. in Form von übertriebener Milde oder Härte bei Bestrafung des Kindes äuBern [9], aber auch in Form eines veränderten, unpassenden Kommunikations- oder Verhaltensstils (z. B. undeutliche oder lautere Sprache, ausgeprägtes Bedürfnis nach körperlicher Zuneigung). Durch die fehlende Konstanz mangelt es für die Kinder an Orientierung. Weitere Auswirkungen der elterlichen Verhaltensvolatilität zeigen sich darin, dass Versprechungen oder Pläne durch einen übermäßigen Konsum hinfällig werden oder spontan geändert werden müssen. Dies kann eine Störung von Familienritualen (wie z. B. gemeinsame Mahlzeiten einzunehmen) mit sich bringen [14], welche für die Kinder eigentlich stabilisierend wirken sollten.

Da ein suchtkrankes Elternteil oftmals nicht in der Lage ist, seine alltäglichen und familiären Verpflichtungen in einem adäquaten Maß wahrzunehmen, werden Verantwortlichkeiten innerhalb der Familie nicht selten neu verteilt. Durch die neuen Rollenverteilungen bekommen die Kinder häufig Aufgaben zugeschrieben, welche nicht altersadäquat sind und geraten in altersunangemessene Muster, vor allem in Bezug auf erwachsenentypische Verpflichtungen. Dieses Phänomen wird als Parentifizierung der Kinder bezeichnet. Dadurch stellt sich für die weitere Entwicklung des Kindes eine Gefahr ein, da die Kinder ihre Entwicklungsaufgaben nicht adäquat bewältigen können und chronisch überfordert sind [15].

Insgesamt erscheinen suchtkranke Eltern wenig in der Lage zu sein, eine positive Atmosphäre im Zusammensein mit dem Kind aufzubauen und verlässlich aufrechtzuerhalten [16], außerdem fördern sie Problemlösefähigkeiten und Durchhaltevermögen des Kindes nur unzureichend. Sie zeigen zudem eine geringe emotionale Responsivität und Feinfühligkeit im Umgang mit dem Kind und seinen Bedürfnissen, sodass sich häufig eine unsichere Bindung zwischen Eltern und Kind entwickelt.

\section{Besonderheiten von Kindern drogenabhängiger Eltern}

Kinder von drogenabhängigen Eltern erleben im Gegensatz zu Kindern alkoholabhängiger Eltern häufiger eine Abhängigkeitserkran- kung bei beiden Elternteilen, da bei Drogenabhängigen ein entsprechendes - subkulturell bedingtes - Partnerwahlverhalten üblicher ist als bei Alkoholabhängigen [17]. Dadurch können die negativen Effekte des drogenabhängigen Elternteils nicht hinreichend durch einen gesunden Elternteil kompensiert werden. Ein möglicher risikominimierender Effekt („Buffering-Effect“) kann dadurch seltener bis gar nicht auftreten. Die höhere Rate an Frühgeburten und das oftmals schwierige Temperament von Kindern drogenabhängiger Mütter kann bei den Eltern Überforderungsgefühle und psychischen Stress auslösen und ihre Beziehung zu den Kindern beeinträchtigen. Betroffene Kinder erleben außerdem die typischen Bedingungen der Drogensubkultur, wie bspw. Beschaffungskriminalität, Prostitution, Strafverfolgung o. ä. [4]. Typisch für Kinder aus Familien mit Drogenproblematik ist außerdem eine stärkere soziale Isolation oder gar Ausgrenzung durch das soziale Umfeld als Folge einer befürchteten oder tatsächlichen gesellschaftlichen Stigmatisierung. Dadurch lernen sie weniger sozial förderliche Verhaltensweisen und erleben sich insgesamt in ihrem Selbstwertgefühl als instabiler und gefährdeter [17]. Durch die vergleichsweise höhere psychische Komorbiditätsrate drogenabhängiger Eltern laufen deren Kinder zudem Gefahr, häufiger eine schwerwiegendere Schädigung zu erleiden. Im Bereich der illegalen Drogen fanden sich auf Elternseite zudem vermehrt justizielle Probleme sowie daraus resultierende Inhaftierungen und damit verbunden häufigere Trennungen von Eltern und Kind [9].

Die Komponenten dysfunktionalen Elternverhaltens infolge von Substanzkonsum und/oder einer Abhängigkeitserkrankung sind multipel und betreffen alle relevanten Verhaltensebenen. Elterliche Drogenabhängigkeit stellt eine Kumulierung dieser Risikovariablen dar.

- Tab. 1 fasst die Auswirkungen des elterlichen Substanzkonsums auf die Erziehungskompetenz auf mehreren Ebenen zusammen [18].

Es muss erwähnt werden, dass sich viele suchtkranke Eltern (zumindest retrospektiv) ihres eigenen ungünstigen Erziehungsverhaltens und dessen negativen Auswirkungen auf die Kinder bewusst sind [19]. Dabei scheinen drogenabhängige Eltern weniger zu be- 
schönigen und zu verzerren als alkoholabhängige Eltern. Dies resultiert in starken Gefühlen von Schuld und Sorge; viele Eltern bezeichnen ihre Kinder als primäres Abstinenzmotiv [9]. Daraus kann eine besondere Chance, aber auch Verantwortung der Suchthilfe abgeleitet werden. Die Abstinenzmotivation der suchtkranken Elternteile sollte genutzt werden, ohne die Kinder für die Veränderung und den Therapieprozess zu funktionalisieren.

\section{Auswirkungen des elterlichen Substanzkonsums für betroffene Kinder}

Substanzbezogene Störungen In internationalen Untersuchungen zu Kindeswohl und Entwicklungspsychopathologie gilt eine elterliche Abhängigkeitserkrankung als besonders negativer Risikofaktor für eine gesunde kindliche Entwicklung [20]. Insbesondere im Hinblick auf die Entwicklung eigener substanzbezogener Störungen werden Kinder aus suchtbelasteten Familien als Hochrisikogruppe betrachtet: Betroffene Kinder besitzen ein 2,4- [21] bis 6-fach [22] erhöhtes Risiko, später selbst einmal eine Abhängigkeitserkrankung zu entwickeln. Begünstigende Faktoren für eine intergenerationale Transmission von suchtbezogenen Störungen sind sowohl genetische und personenbezogene als auch Umweltund Lernfaktoren [15].

Externalisierende und internalisierende Verhaltensauffälligkeiten Es wurde wiederholt belegt, dass Kinder aus suchtbelasteten Familien neben Suchtstörungen auch andere psychische Erkrankungen mit erhöhter Wahrscheinlichkeit entwickeln $[4,15]$. So besteht bei Kindern aus suchtbelasteten Familien z. B. eine erhöhte Auftrittswahrscheinlichkeit für externalisierende Auffälligkeiten, wie Störungen des Sozialverhaltens oder hyperkinetische Störungen [23]. Kinder aus suchtbelasteten Familien sind auch bezüglich der Entwicklung internalisierender Störungsbilder besonders gefährdet: Betroffene Kinder zeigen im Vergleich zu unbelasteten Kindern erhöhte Raten an Depressionen [24] oder Angststörungen [25], sowohl bereits im Kindes- als auch später im Erwachsenenalter. Außerdem besteht für die von elterlicher Sucht betroffenen Kinder ein signifikant erhöhtes Risiko für die Entwicklung von Persönlichkeitsstörungen [17]. Externalisierende als auch internalisierende Störungsbilder gelten darüber hinaus als stabile Prädiktoren für die Entwicklung einer eigenen substanzbezogenen Störung [26]. Insofern ist davon auszugehen, dass frühe Verhaltensauffälligkeiten der Kinder im elterlichen Suchtumfeld, die als stressbedingte Reaktionen und Bewältigungsversuche des dysfunktionalen Erziehungsumfeldes anzusehen sind, langfristig zu relevanten psychischen Störungen führen können.

Die Auswirkungen einer elterlichen Abhängigkeitserkrankung und dem daraus resultierenden, dysfunktionalen Elternverhaltens können für Kinder gravierend sein und sich sowohl im Kindes- als auch im Jugend- oder Erwachsenenalter in Form von einer psychischen Symptombelastung äußern.

\section{Hilfeangebote für Eltern mit Suchterkrankungen zur Förderung der Elternkompetenz}

Die aufgezeigten Ergebnisse zum Erziehungsverhalten suchtkranker Eltern und dessen Auswirkungen auf die Kinder unterstreichen den dringenden Unterstützungsbedarf für betroffene Familien. Obwohl es diverse Programme gibt, die sich direkt an Kinder aus suchtbelasteten Familien richten [20], mangelt es im deutschen Hilfesystem an suchtspezifischen, wissenschaftlich fundierten Programmen, die sich direkt an suchtmittelabhängige Eltern richten. Ein verstärktes Angebot wissenschaftlicher fundierter Elterntrainings ist aber dringend notwendig, da das elterliche Erziehungsverhalten eine zentrale Bedeutung für die Entstehung psychischer Störungen im Kindes- und Jugendalter besitzt [27]. Es bedarf im Hilfesystem des transgenerationalen Blicks, um Familienwelten weniger risikohaft werden zu lassen und Risiken für exponierte Kinder systematisch zu verringern. Dies geschieht am besten mit selektiven Präventionsprogrammen im suchtspezifischen Hilfesystem oder im Bereich der Kinder- und Jugendhilfe, aber außerhalb des Schulsystems, weil dort Stigmatisierung und Diskriminierung drohen.

\section{Bestehende Angebote für suchtmittelabhängige Eltern im deutschsprachigen Raum}

Im deutschsprachigen Raum gibt es eine Vielzahl von evaluierten Gruppentrainings für Eltern, die sich an verschiedene Zielgruppen richtet und unterschiedliche Bereiche der Prävention abdeckt [28]. Trainings speziell für suchtkranke Eltern sind dabei allerdings kaum vorhanden. Das bislang bekannteste Programm ist das Mütter-Unterstützungs-Training (MUT!) [29]. MUT! ist ein Gruppentraining für drogenabhängige, substituierte Mütter zur Förderung der Erziehungskompetenz. MUT! soll bei den Müttern u. a. die Wissenskompetenz über die kindliche Entwicklung und die Handlungskompetenz im Umgang mit dem Kind erweitern. Sie werden außerdem in ihrer Rolle als Mütter gestärkt und sollen erziehungsspezifische Selbstwirksamkeit aufbauen. Die Mütter fühlten sich nach Ende des Kurses weniger sozial isoliert, erfuhren eine Verbesserung im Kompetenzerleben sowie einen Rückgang an sozialer Unsicherheit und dysfunktionalen Denkmustern.

Für Männer mit einer Alkoholabhängigkeit existiert das manualisierte Gruppenprogramm Männlichkeiten und Sucht [30]. Diese Intervention richtet sich nicht speziell an Männer mit Kindern, widmet aber ein Modul dem Thema Vaterschaft. Inhaltlich werden die Folgen einer Alkoholabhängigkeit auf das Erziehungsverhalten thematisiert, z. B. häusliche Gewalt. Ziel ist es, die eigene Vaterrolle zu reflektieren, das Zusammensein mit dem Kind zu verbessern und die Männer für die Perspektive ihrer Kinder zu sensibilisieren. Spezifischere Programme, die das Thema „Sucht und Vaterschaft“ fokussieren, sind notwendig, insbesondere da die Mehrzahl der suchtkranken Elternteile Väter sind.

Derzeit erprobt wird das SHIFT-Elterntraining (,Suchthilfe und Familientraining“). SHIFT ist ein modularisiertes, verhaltenstherapeutisches Gruppenprogramm speziell für methamphetaminabhängige Eltern mit Kindern zwischen 0 und 8 Jahren, welches darauf abzielt, die Elternkompetenzen und die familiäre Widerstandsfähigkeit zu verbessern und zu stärken [31]. Gleichzeitig sollen die elterliche Substanzab- 
stinenz stabilisiert und weitere Inanspruchnahmen von Hilfen gefördert werden. Erste Evaluationsergebnisse werden 2018 erwartet.

In Deutschland gibt es kaum wissenschaftlich fundierte Interventionen, die sich speziell an suchtmittelabhängige Mütter und Väter richten. Aufgrund der besonderen Bedeutung der direkten Förderung von Elternkompetenzen, sind Forschung und Praxis jedoch für dieses Thema sensibilisiert und erste Projekte wurden initiiert.

\section{Angebote für suchtmittelabhängige Eltern im US-amerikanischen Raum}

Im US-amerikanischen Raum gibt es bereits verschiedene evidenzbasierte Elterntrainings, die speziell auf die Zielgruppe suchtmittelabhängiger Eltern zugeschnitten sind. Eine aktuelle Übersichtsarbeit [4] befasste sich mit evidenzbasierten Interventionen speziell für Eltern mit Substanzmittelabhängigkeit und identifizierte dabei folgende Programme:

In Parents under Pressure (PUP) lernen teilnehmende Eltern, wie sie die Kindeserziehung gestalten und eine stabile Beziehung mit dem Kind etablieren können. Außerdem werden die elterliche Emotionsregulation verbessert und Anti-Rückfallstrategien erarbeitet. Ergebnisse zur Wirksamkeit von PUP zeigen auf, dass die Intervention das Funktionsniveau der Familie erhöhen und die Wahrscheinlichkeit einer Kindesmisshandlung signifikant verringern konnte. Die psychische Symptombelastung der Kinder konnte signifikant reduziert werden.

Focus on Families (FOF) (jetzt: Families Facing the Future) ist ein Gruppentraining für Eltern im Methadonprogramm. FOF zielt darauf ab, den elterlichen Substanzkonsum zu reduzieren, innerfamiliäre Kommunikationsmuster und Verhaltensweisen zu verbessern sowie Familienkonflikte und Verhaltensprobleme des Kindes zu verringern. FOF erwies sich mittelfristig als effektiv in Hinblick auf eine Reduktion des elterlichen Substanzkonsums und ein verbessertes Erziehungsverhalten. Langfristig hatten Söhne der teilnehmenden Eltern ein geringeres Risiko, selbst eine substanzbezogene Störung zu entwickeln.

Behavioural Couples Therapy (BCT) ist eine Intervention für Männer mit Alkohol- oder Drogenproblemen, welche effektive Kommunikationsstrategien, eine positive Reziprozität sowie Strategien zur Eliminierung verbaler und physischer Aggression zwischen (Ehe-) Partnern vermittelt. Evaluationsergebnisse belegen, dass das psychosoziale Funktionsniveau der Kinder durch die Teilnahme der Eltern signifikant verbessert werden konnte. Das Zufügen einer Eltern-Komponente war der ursprünglichen Fassung der BCT in Form einer weiteren reduzierten psychischen Symptombelastung der Kinder zudem überlegen.

Ein neueres systematisches Review [32] identifizierte neben den bereits genannten weitere evidenzbasierte Interventionen für suchtmittelabhängige Eltern. Der Fokus lag dabei auf Interventionen, die hauptsächlich kognitiv-behaviourale Elemente beinhalten (verhaltensorientiert, „skill-based programs“). Hierzu gehört z. B. das auch in Deutschland an mehreren Standorten angebotene Strengthening Families Program (SFP) (deutsch: Familien stärken) [33]. SFP richtet sich speziell an Risikofamilien und beinhaltet Eltern-, Kinder- und Familiensitzungen. In den Elternsitzungen geht es um die Demonstration von Liebe und positiver Verstärkung unter gleichzeitiger Berücksichtigung des Setzens von Grenzen und Aufstellens von Regeln. Weitere Inhalte sind Stress- und Konfliktbewältigung. SFP konnte das Elternverhalten positiv verändern, die Kommunikation verbessern sowie den elterlichen Substanzgebrauch, depressive Symptome, körperliche Gewalt und kindliche Verhaltensauffälligkeiten signifikant reduzieren. Die größten Effektstärken wurden dabei für Familien mit Kindern zwischen 6 und 11 Jahren gefunden. Von SFP existieren verschiedene Varianten [32]: So besteht bspw. mit Parenting While Incarcerated (PWI) eine Adaption für inhaftierte Eltern mit Suchtproblemen.

Verhaltensbasierte Elternprogramme sind auch bei Eltern mit einer Abhängigkeitserkrankung wirksam. Der Erfolg der Programme liegt in der effektiven Vermittlung von elterlichen Verhaltensstrategien, die direkt das Verhalten des Kindes beeinflussen können.

An verhaltensorientierten Elternprogrammen wird kritisiert, dass sie ausschließlich das beobachtbare Verhalten der Eltern in Augenschein nehmen und dabei die emotionale Beziehungs- und damit auch Bindungsqualität zwischen Eltern und Kind relativ unbeachtet lassen [32, 34]. Dabei zeigen Ergebnisse der Bindungsforschung, dass die emotionale Qualität der Eltern-Kind-Beziehung einen der wichtigsten Prädiktoren für die gesunde psychische Entwicklung von Kindern in allen Altersstufen darstellt [34]. Das o.g. Review [32] bezieht demgemäß auch Programme ein, die insbesondere die Eltern-Kind-Beziehung und Bindung in Fokus nehmen (,attachment-based programs“):

Das Mothers and Toddlers Program (MTP) fokussiert explizit das Thema Bindung im Kontext von Abhängigkeitserkrankungen. Das Programm wird als Ergänzung zur traditionellen Suchtbehandlung eingesetzt und wurde entwickelt, um das Fürsorgeverhalten der Eltern zu verbessern. Hierzu soll vor allem die Reflektions- und Mentalisierungsfähigkeit, die Emotionsregulation und Sensitivität und Handlungskompetenz der Mutter gegenüber ihrem Kind gestärkt werden. Das MTP erwies sich als wirksam in Bezug auf eine Reduktion der elterlichen depressiven Symptomatik, Drogenabstinenz und ein verbessertes Fürsorgeverhalten.

Im Programm Attachment and Biobehavioural Catch-up (ABC) steht die Förderung der elterlichen Fürsorge und Interaktion zwischen Eltern und Kind bei Hochrisikogruppen im Fokus. ABC basiert im Wesentlichen auf Beobachtungen der Interaktionen der ElternKind-Dyade, welche auf Video aufgenommen und anschließend gemeinsam analysiert werden. Erste Ergebnisse demonstrieren einen Effekt von $A B C$ bezüglich eines fürsorglicheren und unterstützenden Elternverhaltens.

Die Circle of Security Parenting Intervention (CoS) zielt darauf ab, die Eltern darin zu stärken, die Signale des Kindes besser zu erkennen, die eigene Observationsfähigkeit und entsprechende Schlussfolgerungen zu verbessern sowie die eigene Emotionsregulation, Reagibilität und die Mentalisierungsfähigkeit zu fördern. Vorläufige Befunde belegen, dass teilnehmende Mütter am Ende des Programms sensibler auf ihre Kinder reagierten und die Kinder ein sichereres Bindungsverhalten aufwiesen.

Cherish the Family (CTF) wurde speziell für suchtbelastete Familien entwickelt, in der das Risiko einer Inobhutnahme des Kindes 
- Tab. 2 Verhaltens- und bindungsorientierte Interventionen für suchtmittelabhängige Eltern, geordnet nach Altersgruppe des Kindes.

\begin{tabular}{|c|c|c|c|c|}
\hline Name Programm & $\begin{array}{l}\text { Alter des } \\
\text { Kindes }\end{array}$ & Zielgruppe & Modus & Setting \\
\hline \multicolumn{5}{|l|}{$\begin{array}{l}\text { Baby- }(0-3 \mathrm{~J} .) \text { und/oder Kleinkind- } \\
\text { alter (3-6].) }\end{array}$} \\
\hline $\begin{array}{l}\text { Attachment and Biobehavioural } \\
\text { Catch-up (ABC) }\end{array}$ & $0-2$ & Hochrisikofamilien & Familie & home-based \\
\hline $\begin{array}{l}\text { Mothers and Toddlers Program } \\
\text { (MTP) }\end{array}$ & $0-3$ & $\begin{array}{l}\text { Mütter mit Drogen- oder Alkohol- } \\
\text { abhängigkeit }\end{array}$ & Einzel & home-based \\
\hline Cherish the Family (CTF) & $0-3$ & $\begin{array}{l}\text { Mütter mit Drogen- oder Alkohol- } \\
\text { abhängigkeit oder HIV/AIDS }\end{array}$ & Einzel & home-based \\
\hline MUT! & $0-6$ & Mütter in Substitutionsbehandlung & Gruppe & center-based \\
\hline SHIFT-Elterntraining & $0-8$ & Eltern mit Methamphetaminkonsum & Gruppe & center-based \\
\hline $\begin{array}{l}\text { Circle of Security Parenting } \\
\text { Intervention (CoS) }\end{array}$ & $1-4$ & $\begin{array}{l}\text { Eltern mit Risiko/erfolgte Inobhut- } \\
\text { nahme des Kindes }\end{array}$ & Gruppe/Einzel & home-/center-based \\
\hline Parents under Pressure (PUP) & $3-8$ & $\begin{array}{l}\text { Eltern mit Drogen- oder Alkoholab- } \\
\text { hängigkeit/in Substitutionsbehand- } \\
\text { lung }\end{array}$ & Einzel/Elternpaar & home-based \\
\hline \multicolumn{5}{|l|}{$\begin{array}{l}\text { Frühes und spätes Schulkindalter } \\
\text { (6-13 Jahre) }\end{array}$} \\
\hline $\begin{array}{l}\text { Focus on Families (FOF) (Families } \\
\text { Facing the Future) }\end{array}$ & $3-14$ & Eltern in Substitutionsbehandlung & Gruppe/Einzel & home-/center-based \\
\hline $\begin{array}{l}\text { Strengthening Families Program } \\
\text { (SFP) }\end{array}$ & $10-14$ & Hochrisikofamilien & Einzel/Familie & home-based \\
\hline \multicolumn{5}{|l|}{ Gesamte Kindheit und Jugend } \\
\hline MATRIX & - & $\begin{array}{l}\text { Klienten mit Methamphetaminabhän- } \\
\text { gigkeit und Angehörige }\end{array}$ & Gruppe & center-based \\
\hline $\begin{array}{l}\text { Parenting while Incarcerated (PWI) } \\
\text { (Adaption von SFP) }\end{array}$ & - & $\begin{array}{l}\text { Inhaftierte Frauen aus Hochrisikofa- } \\
\text { milien }\end{array}$ & Gruppe & center-based \\
\hline Behavioural Couples Therapy (BCT) & - & $\begin{array}{l}\text { Männer mit Drogen- oder Alkoholab- } \\
\text { hängigkeit }\end{array}$ & Einzel/Paar & center-based \\
\hline
\end{tabular}

oder eines Sorgerechtsentzugs besteht oder bereits erfolgt ist. CTF konzentriert sich neben der Verbesserung der Eltern-Kind-Bindung auf den Aufbau der Inanspruchnahme von Hilfen sowie auf die Förderung der Stressbewältigung und körperlichen Gesundheit bei den Eltern. Erste Evaluationsergebnisse mit inhaftierten Müttern belegen, dass $45 \%$ der Teilnehmerinnen vermehrt Hilfen in Anspruch nahmen, verbesserte Interaktionsfähigkeiten und Elternkompetenzen demonstrierten und weniger Ambivalenz hinsichtlich einer Rückführung des Kindes aufzeigten.

Bindungsorientierte Elterntrainings sind in der internationalen Forschung zunehmend in den Fokus gerückt und erscheinen erfolgversprechend, da sie direkt auf die Verbesserung der Eltern-Kind-Beziehung abzielen.

\section{Substanzspezifische Interventionen für suchtkranke Eltern}

Allen genannten Programmen ist gemein, dass sie substanzübergreifend alle Eltern integrieren, die Probleme mit illegalen Drogen und/ oder Alkohol haben. Für spezielle Subgruppen von Konsumenten ist vor allem das MATRIX-Programm hervorzuheben [35]. MATRIX ist ein ambulantes, verhaltensorientiertes Behandlungsprogramm für Methamphetaminabhängige. Für dieses Programm bestehen ergänzende Zusatzmaterialien, die das Thema Familie speziell in den Fokus nehmen, wie z. B. das Beiheft zur Behandlung von weiblichen Klientinnen (Using MATRIX with Women Clients) [36]. Hier werden im Rahmen von zusätzlichen Gruppensitzungen Aspekte behandelt, die für den Genesungsprozess von Konsumentinnen wichtig sein können, wie z. B. Schwangerschaft und Elternschaft. Inhaltliche Schwerpunkte liegen dabei u. a. auf der Vermittlung von Erziehungsfertigkeiten, Psychoedukation, Bearbeitung von Schuldgefühlen sowie Sexualität und Schwangerschaft im Kontext von Crystal Meth.

Im US-amerikanischen Raum existieren verschiedene evidenzbasierte und effektive verhaltens- und bindungsorientierte Interventionen, die speziell Eltern mit Suchterkrankungen adressieren. Dabei sind die Inhalte der Trainings sowohl substanzunspezifisch als auch substanzspezifisch aufbereitet. 
- Tab. 2 stellt die genannten Verhaltens- und bindungsorientierte Interventionen für suchtmittelabhängige Eltern geordnet nach der hauptsächlicher Altersgruppe des Kindes in einer Übersicht dar und beinhaltet weiterführende Informationen zum Modus und Setting.

\section{FAZIT FÜR DIE PRAXIS}

Die Auswirkungen einer elterlichen Abhängigkeitserkrankung und dem resultierenden, dysfunktionalen Elternverhalten können für Kinder schwerwiegende negative Konsequenzen haben. Dabei ist zu denken an frühkindliche Entwicklungsstörungen und/oder psychische Störungen im Kindes-, Jugend- oder Erwachsenenalter. Forschungsergebnisse zu Interventionen für suchtmittelabhängige Eltern haben gezeigt, dass spezifische Elterntrainings durch die Förderung der Eltern-und Erziehungskompetenzen der betroffenen Eltern die psychosoziale Situation ihrer Kinder signifikant verbessern können. Als besonders effektiv haben dabei sich sowohl verhaltens- als auch bindungsorientierte Elemente erwiesen.

Für suchtkranke Eltern in ihrer krankheitstypischen Ambivalenz in Bezug auf Abstinenz und Veränderung kann die Reflexion des Kindeswohls und die Perspektive einer gelingenden Elternschaft eine wichtige Motivation für Abstinenz und Therapie darstellen. Dabei sollte die Rolle der Elternschaft in der Therapieplanung und -durchführung berücksichtigt werden, da es sich um ein zentrales Thema des Alltags betroffener Mütter, Väter und Kinder handelt und darüber hinaus ein besonders emotionsgenerierender Bereich angesprochen wird. Dies ist auch im Sinne der Wirkfaktoren der Psychotherapie besonders aussichtsreich, da besonders die Bereiche „Problemaktualisierung“ und „aktive Hilfe zur Problembewältigung“ angesprochen werden [37]. Aus einer transgenerationalen Kindperspektive sind elternbezogene Hilfen eine potentielle Prävention von Fehlentwicklungen und psychischen Störungen bei den Kindern und können eine sinnvolle Ergänzung direkter kindbezogener Präventionsmaßnahmen darstellen. Insofern bleibt zu wünschen, dass sich auch die immer mehr auf Erwerbsfragen bezogene Suchtrehabilitation abhängigkeitskranker Eltern den transgenerationalen Bedürfnissen von suchtbelasteten Familien öffnet.

\section{Über die Autoren}

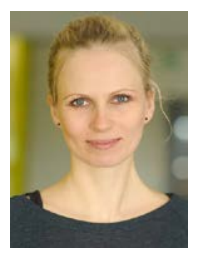

\section{Dr. rer. nat. Diana Moesgen}

Psychologin, M.Sc., Psych. Psychotherapeutin (VT). Seit 2006 wissenschaftliche Mitarbeiterin am Deutschen Institut für Sucht- und Präventionsforschung der Katholischen Hochschule NRW in Köln, seit 2016 nebenberuflich in eigener Praxis tätig.

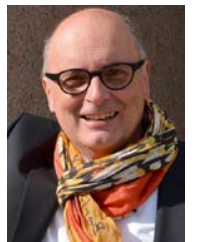

\section{Prof. Dr. rer. nat. Michael Klein}

Dipl.-Psychologe, Psych. Psychotherapeut (VT). Seit 1994 Professor für Klinische Psychologie und Suchtforschung an der Katholischen Hochschule NRW, Köln, Leiter des dortigen Deutschen Instituts für Sucht- und Präventionsforschung.

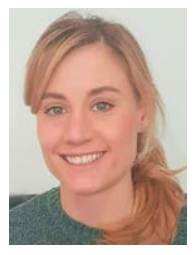

\section{Dipl.-Psych. Janina Dyba}

Seit 2014 als wissenschaftliche Mitarbeiterin am Deutschen Institut für Sucht- und Präventionsforschung der Katholischen Hochschule NRW.

Interessenskonflikt

Die Autoren geben an, dass kein Interessenskonflikt besteht.

\section{Literatur}

[1] European Monitoring Centre for Drugs and Drug Addiction (EMCDDA). Drugs and vulnerable groups of young people. Selected Issue. Lissabon: EMCDDA; 2008

[2] Robert Koch Institut. Beiträge zur Gesundheitsberichterstattung des Bundes. Daten und Fakten: Ergebnisse der Studie "Gesundheit in Deutschland aktuell 2012”. Berlin: Robert Koch Institut; 2014

[3] NACOA Deutschland. Kinder aus suchtbelasteten Familien. Hilfen zur Unterstützung in Kindertagesstätte und Grundschule. Berlin: NACOA; 2006

[4] Calhoun S, Conner E, Miller M et al. Improving the outcomes of children affected by parental substance abuse: a review of randomized controlled trials. Subst Abuse Rehabil 2015; 6: 15-24

[5] Landgraf MN, Heinen F. Fetales Alkoholsyndrom. Stuttgart: Kohlhammer; 2013

[6] Drogenbeauftragte der Bundesregierung, (Hrsg.). S3-Leitlinie Methamphetaminbezogene Störungen. Heidelberg: Springer; 2016

[7] Lester BM, Lagasse LL. Children of addicted women. Journal of Addictive Diseases 2010; 29: 259-276

[8] Hussong AM, Flora DB, Curran PJ et al. Defining risk heterogeneity for internalizing symptoms among children of alcoholic parents. Develop Psychopathol 2008; 20: 165-193

[9] Klein M, Dyba J, Moesgen D. Crystal meth und familie - Zur Analyse der Lebenssituation und des Hilfebedarfs betroffener Kinder. Berlin: Bundesministerium für Gesundheit; 2016

[10] Hogan DM. The impact of opiate dependence on parenting processes: Contextual, physiological and psychological factors. Addiction Research \& Theory 2007; 15: 617-635

[11] Petermann F, Petermann U. Erziehungskompetenz. Kindheit und Entwicklung 2006; 15: 1-8

[12] Olszewski D, Burkhart G, Bo A. Children's voices. Experiences and perceptions of European children on drug and alcohol issues. Lissabon: EMCDDA; 2010

[13] Templeton L, Velleman R, Hardy E et al. Young People living with parental alcohol misuse and parental violence: "No-one has ever asked me how I feel in any of this". Journal of Substance Use 2009; 14: $139-150$ 
[14] Zobel M. Kinder aus alkoholbelasteten Familien. Entwicklungsrisiken und -chancen. Göttingen: Hogrefe; 2000

[15] Moesgen D. Kinder und Jugendliche aus alkoholbelasteten Familien. Dysfunktionale Kognitionen und Verhaltensauffälligkeiten. Hamburg: disserta; 2014

[16] Belsky J, Jeffee SR. The Multiple Determinants of Parenting. In: Cicchetti D, Cohen DJ. Developmental Psychopathology. Risk, Disorder, and Adaptation. Vol. 3: 2nd Ed.Hoboken, NJ: Wiley \& Sons; 2006: 38-85

[17] Klein M. Kinder aus alkoholbelasteten Familien. In Klein M, (Hrsg.). Kinder und Suchtgefahren. Stuttgart: Schattauer; 2008: 114-127

[18] Hilbig K. Crystal - Auswirkungen auf Erziehungsverantwortung und Erziehungskompetenz. Vortrag auf dem Fachtag „Crystal - Vater, Mutter, Kind“, Dresden. 2013

[19] Haight WL. Children of methamphetamine-involved families. The case of rural Illinois. Oxford, NY: Oxford University Press; 2009

[20] Klein M, Moesgen D, Bröning $S$ et al. Kinder aus suchtbelasteten Familien stärken. Das „Trampolin“-Programm. Göttingen: Hogrefe 2013

[21] Ulrich I, Stopsack M, Barnow S. Risiko- und Resilienzfaktoren von adoleszenten Kindern alkoholkranker Eltern. Ergebnisse der Greifswalder Familienstudie. Diskurs Kindheits- und Jugendforschung 2010; 5: 47-61

[22] Grant BF. Estimates of U.S. children exposed to alcohol abuse and dependence in the family. Am J Public Health 2000; 90: 112-115

[23] Kendler KS, Ohlsson H, Sundquist K et al. Cross-generational transmission from drug abuse in parents to attention-deficit/ hyperactivity disorder in children. Psychol Med 2016; 46: 1301

[24] Fuller-Thomson E, Katz BR, Phan TV et al. The long arm of parental addictions: the association with adult children's depression in a population-based study. Psychiatry Res 2013; 210: 95-101

[25] MacPherson PSR, Stewart SH, McWilliams LA. Parental problem drinking and anxiety disorder symptoms in adult offspring. Examining the mediating role of anxiety sensitivity components. Addictive Behaviours 2001; 26: 917-934

[26] King KM, Chassin L. Adolescent Stressors, Psychopathology, and Young Adult Substance Dependence: A Prospective Study. J Stud Alcohol 2008; 69: 629-638
[27] Petermann F. Elternberatung und Elterntraining. In: Linden M, Hautzinger M, (Hrsg.). Verhaltenstherapiemanual. 7. AuflHeidelberg: Springer; 2011: 383-388

[28] Bundeszentrale für gesundheitliche Aufklärung (BZgA). Expertise Gesundheitsfördernde Elternkompetenzen für das frühe Kindesalter. Köln: Bundeszentrale für gesundheitliche Aufklärung; 2011

[29] Klein M. Kinder drogenabhängiger Eltern. Risiken, Fakten, Hilfen. In: Klein M, Hoff T, Pauly A. Schriftenreihe Angewandte Suchtforschung (Band 2). Regensburg: Roderer; 2006

[30] Landschaftsverband Westfalen-Lippe. Anwendung und Bewertung des Handbuchs „Männlichkeiten und Sucht“ - Eine Evaluationsstudie. Forum Sucht 2014; 9: 1-89

[31] Moesgen D, Klein M, Dyba J. Konzeption einer Intervention für methamphetaminabhängige Eltern zur Förderung der Familienresilienz und Elternkompetenz - Ergebnisse der Manualentwicklung. Vortrag auf dem Deutschen Suchtkongress 2016

[32] Renk K, Boris NW, Kolomeyer E et al. The state of evidence-based parenting interventions for parents who are substance-involved. Pediatr. Res. 2016; 79: 177-183

[33] Bröning S, Sack PM, Thomsen M et al. Kinder mit multipler Risikoexposition profitieren von der Teilnahme an „Familien stärken!“. Prax Kinderpsychol Kinderpsychiatr 2016; 65: 550-566

[34] Suchman N, Mayes L, Conti J et al. Rethinking parenting interventions for drug-dependent mothers: From behaviour management to fostering emotional bonds. J Subst Abuse Treat 2004; 27 : 179-185

[35] Center for Substance Abuse Treatment. Counselor's Treatment Manual: Matrix Intensive Outpatient Treatment for People With Stimulant Use Disorders. DHHS Publication No. (SMA) 07-4152. Rockville, MD: Substance Abuse and Mental Health Services Administration; 2007

[36] Substance Abuse and Mental Health Services Administration (SAMHSA). Using Matrix with Women Clients: A Supplement to the Matrix Intensive Outpatient Treatment for People with Stimulant Use Disorders. HHS Pub. No. (SMA) 12-4698. Rockville, MD: Substance Abuse and Mental Health Services Administration; 2012

[37] Grawe K, Grawe-Gerber M. Ressourcenaktivierung. Ein primäres Wirkprinzip der Psychotherapie. Psychotherapeut 1999; 44: 63-73 